\title{
A cost-optimal parallel tridiagonal system solver
}

\author{
Ferng-Ching LIN and Kuo-Liang CHUNG \\ Department of Computer Science and Information Engineering, National Taiwan University, Taipei, \\ Taiwan 10764, R.O.C.
}

Received September 1989

Revised February 1990

\begin{abstract}
We first show how to transform the solution of an $n \times n$ tridiagonal system into suffix computations of continued fractions. Then a parallel substitution scheme is introduced to compute the suffix values. The derived parallel algorithm allows the tridiagonal system to be solved in $O(\log n)$ time on an unshuffle network with $\Theta(n / \log n)$ processors. It is cost-optimal in the sense that processor number times execution time is minimized. Our solver is conceptually simple and easy for implementation.
\end{abstract}

Keywords. Continued fraction, Suffix values, Recurrence equations, Substitution scheme, Tridiagonal system, Unshuffle.

\section{Introduction}

Consider a system of linear equations $A x=b$, where the $n \times n$ coefficient matrix $A$ is tridiagonal, that is, only elements directly on, directly above, and directly below the diagonal are nonzero and all the other entries are zero. The solution of a tridiagonal system lies in the heart of many important problems, such as the spline interpolation by cubic polynomials and the solutions of partial differential equations. It is in fact very easy to derive a sequential method for solving the tridiagonal systems in $O(n)$ time [7].

Based on the LU decomposition of matrix $A$, Stone $[17,18]$ first proposed a recursive-doubling method to solve the system in $O(\log n)$ time using $\Theta(n)$ processors in parallel. Sameh and Kuck [16] showed that by the use of QR factorization the system can be solved in $O(\log n)$ time with $\Theta(n)$ processors too. The odd-even reduction method, which was due to Hockney [10], also provides parallel algorithms with the same cost [9,13]. Wang [20] presented a divide-and-conquer method which has the same cost and is suitable for both parallel and vector computers. Recently, the odd-even reduction method is applied to solve the tridiagonal system on the perfect shuffle network [11]. The time needed is $O(\log n)$ and the number of processors used is $n /(1+\alpha)$, where $\alpha=$ (the time to communicate one floating-point number) $/$ (the time to perform one floating-point arithmetic operation).

In this paper, we first show that the solution of a tridiagonal system can be converted into continued fraction based computations which correspond to the Gaussian elimination and the back-substitution applied on the system. Then, a parallel substitution scheme is introduced to compute the suffix values of the continued fractions. The derived parallel algorithm allows the tridiagonal system to be solved in $O(\log n)$ time on a network in which $\Theta(n / \log n)$ processors communicate through an unshuffle routing mechanism. Our solver greatly reduces the number of processors used previously and is cost-optimal in the sense that processor number times execution time is minimized. 


\section{Transformation to continued fractions}

Suppose we have the following tridiagonal system:

$$
\begin{aligned}
& d_{1} x_{1}+e_{1} x_{2}=b_{1}, \\
& c_{i} x_{i-1}+d_{i} x_{i}+e_{i} x_{i+1}=b_{i} \text { for } i=2,3, \ldots, n-1, \\
& c_{n} x_{n-1}+d_{n} x_{n}=b_{n} .
\end{aligned}
$$

Assuming $d_{1} \neq 0$, we can eliminate $x_{1}$ from the second equation to get the new equation

$$
d_{2}^{\prime} x_{2}+e_{2} x_{3}=b_{2}^{\prime}
$$

where $d_{2}^{\prime}=d_{2}-c_{2} e_{1} / d_{1}$ and $b_{2}^{\prime}=b_{2}-c_{2} b_{1} / d_{1}$. Next, we use the above equation to eliminate $x_{2}$ from the third equation (assuming $d_{2}^{\prime} \neq 0$ ) and get

$$
d_{3}^{\prime} x_{3}+e_{3} x_{4}=b_{3}^{\prime} \text {, }
$$

where $d_{3}^{\prime}=d_{3}-c_{3} e_{2} / d_{2}^{\prime}$ and $b_{3}^{\prime}=b_{3}-c_{3} b_{2}^{\prime} / d_{2}^{\prime}$. In general, at stage $(k-1), 2 \leqslant k \leqslant n$, we eliminate $x_{k-1}$ from the $k$ th equation (assuming $d_{k-1}^{\prime} \neq 0$ ) and get

$$
d_{k}^{\prime} x_{k}+e_{k} x_{k+1}=b_{k}^{\prime} \text {, }
$$

with

$$
d_{k}^{\prime}=d_{k}-c_{k} e_{k-1} / d_{k-1}^{\prime}
$$

and

$$
b_{k}^{\prime}=b_{k}-c_{k} b_{k-1}^{\prime} / d_{k-1}^{\prime} \text {. }
$$

This procedure is the well-known Gaussian elimination.

During the back-substitution for obtaining $x_{i}, i=n, n-1, \ldots, 1$, we first have (assuming $d_{n}^{\prime} \neq 0$ )

$$
x_{n}=\frac{b_{n}^{\prime}}{d_{n}^{\prime}}
$$

and then

$$
x_{k}=\frac{b_{k}^{\prime}-e_{k} x_{k+1}}{d_{k}^{\prime}}
$$

for $k=n-1, n-2, \ldots, 1$.

Up to here, the solution of the tridiagonal system has been transformed into three recurrence equations, namely, (2.1), (2.2) and (2.3), for $d_{k}^{\prime}, b_{k}^{\prime}$ and $x_{k}$ respectively. In the remainder of this section, these recurrences will be further transformed into suffix computations of continued fractions.

Notation. The following form represents a finite continued fraction (CF):

$$
l_{1}+\frac{m_{2}}{l_{2}+\frac{m_{3}}{\ldots \frac{l_{n-1}+\frac{m_{n}}{l_{n}}}{l_{n}}}}
$$

Such a representation for CF is typographically cumbersome and can be replaced by a more compact form:

$$
l_{1}+\frac{m_{2}}{l_{2}+} \frac{m_{3}}{l_{3}+} \cdots \frac{m_{n}}{l_{n}}
$$


Let us define a second-order linear recurrence:

$$
\begin{aligned}
& f_{0}=1, \quad f_{1}=d_{1}, \\
& f_{k}=d_{k} f_{k-1}-c_{k} e_{k-1} f_{k-2} \quad \text { for } k \geqslant 2 .
\end{aligned}
$$

It is easily seen that $d_{k}^{\prime}=f_{k} / f_{k-1}$ for $k \geqslant 1$. The computation of $d_{k}^{\prime}$ breaks down when $f_{k-1}=0$. Nevertheless, this rarely happens in practice although there is no theoretical guarantee for it [1].

By (2.4), $d_{n}^{\prime}=f_{n} / f_{n-1}=\left(d_{n} f_{n-1}-c_{n} e_{n-1} f_{n-2}\right) / f_{n-1}=d_{n}+\left(-c_{n} e_{n-1}\right) /\left(f_{n-1} / f_{n-2}\right)$. So $d_{n}^{\prime}$ can be expressed as the following CF:

$$
d_{n}^{\prime}=d_{n}+\frac{-c_{n} e_{n-1}}{d_{n-1}+} \frac{-c_{n-1} e_{n-2}}{d_{n-2}+} \cdots \frac{-c_{3} e_{2}}{d_{2}+} \frac{-c_{2} e_{1}}{d_{1}} \text {. }
$$

We may assume that $n$ is a power of 2 ; otherwise, pad $d_{n}^{\prime}$ with a special CF

$$
\frac{0}{1+} \frac{0}{1+} \cdots \frac{0}{1}
$$

to enlarge $n$ to be a power of 2 without altering the computation results.

The $k$ th suffix value of $d_{n}^{\prime}$ is $d_{k}^{\prime}$, i.e.

$$
d_{k}^{\prime}=d_{k}+\frac{-c_{k} e_{k-1}}{d_{k-1}+} \frac{-c_{k-1} e_{k-2}}{d_{k-2}+} \cdots \frac{-c_{3} e_{2}}{d_{2}+} \frac{-c_{2} e_{1}}{d_{1}} \text {. }
$$

Obviously, $d_{k}^{\prime}$ can be sequentially converted into its rational form in $O(k)$ time. Our goal is to speed up the computation of obtaining the rational form of $d_{k}^{\prime}$ for all $k=1,2, \ldots, n$.

\section{The parallel substitution scheme}

Let the rational form $\left(r_{1}+r_{2} d_{1}^{\prime}\right) /\left(r_{3}+r_{4} d_{i}^{\prime}\right)$ be represented by $\left(r_{1}, r_{2}, r_{3}, r_{4}, d_{i}^{\prime}\right)$. Here $d_{i}^{\prime}$ can be considered as a pseudo variable for substitution. The CF of, say, $d_{8}^{\prime}$ can be decomposed into 8 sub_CFs:

$$
\begin{aligned}
& d_{8}^{\prime}=d_{8}+\frac{-c_{8} e_{7}}{d_{7}^{\prime}}=\left(-c_{8} e_{7}, d_{8}, 0,1, d_{7}^{\prime}\right), \\
& d_{7}^{\prime}=d_{7}+\frac{-c_{7} e_{6}}{d_{6}^{\prime}}=\left(-c_{7} e_{6}, d_{7}, 0,1, d_{6}^{\prime}\right), \\
& \vdots \\
& d_{3}^{\prime}=d_{3}+\frac{-c_{3} e_{2}}{d_{2}^{\prime}}=\left(-c_{3} e_{2}, d_{3}, 0,1, d_{2}^{\prime}\right), \\
& d_{2}^{\prime}=d_{2}+\frac{-c_{2} e_{1}}{d_{1}^{\prime}}=\left(-c_{2} e_{1}, d_{2}, 0,1, d_{1}^{\prime}\right), \\
& d_{1}^{\prime}=\frac{d_{1}}{1+d_{0}^{\prime}}=\left(d_{1}, 0,1,1, d_{0}^{\prime}\right) .
\end{aligned}
$$

The pseudo-variable $d_{0}^{\prime}$ will be replaced by zero after all the substitutions are completed.

Since substituting $d_{i}^{\prime}=\left(s_{1}+s_{2} d_{j}^{\prime}\right) /\left(s_{3}+s_{4} d_{j}^{\prime}\right)$ into $\left(r_{1}+r_{2} d_{i}^{\prime}\right) /\left(r_{3}+r_{4} d_{i}^{\prime}\right)$ gives $\left(\left(r_{1} s_{3}+\right.\right.$ $\left.\left.r_{2} s_{1}\right)+\left(r_{1} s_{4}+r_{2} s_{2}\right) d_{j}^{\prime}\right) /\left(\left(r_{3} s_{3}+r_{4} s_{1}\right)+\left(r_{3} s_{4}+r_{4} s_{2}\right) d_{j}^{\prime}\right)$, the substitution operation which uses 8 multiplications and 4 additions can be defined as:

$$
\begin{aligned}
& \left(r_{1}, r_{2}, r_{3}, r_{4}, d_{i}^{\prime}\right) \circ\left(s_{1}, s_{2}, s_{3}, s_{4}, d_{j}^{\prime}\right) \\
& \quad=\left(r_{1} s_{3}+r_{2} s_{1}, r_{1} s_{4}+r_{2} s_{2}, r_{3} s_{3}+r_{4} s_{1}, r_{3} s_{4}+r_{4} s_{2}, d_{j}^{\prime}\right) .
\end{aligned}
$$


Lemma 3.1. The substitution operation $\circ$ is associative.

Proof. For any $\left(r_{1}, r_{2}, r_{3}, r_{4}, d_{i}^{\prime}\right),\left(s_{1}, s_{2}, s_{3}, s_{4}, d_{j}^{\prime}\right)$ and $\left(t_{1}, t_{2}, t_{3}, t_{4}, d_{k}^{\prime}\right)$, we have

$$
\begin{aligned}
& {\left[\left(r_{1}, r_{2}, r_{3}, r_{4}, d_{i}^{\prime}\right) \circ\left(s_{1}, s_{2}, s_{3}, s_{4}, d_{j}^{\prime}\right)\right] \circ\left(t_{1}, t_{2}, t_{3}, t_{4}, d_{k}^{\prime}\right) } \\
&=\left(r_{1} s_{3}+r_{2} s_{1}, r_{1} s_{4}+r_{2} s_{2}, r_{3} s_{3}+r_{4} s_{1}, r_{3} s_{4}+r_{4} s_{2}, d_{j}^{\prime}\right) \circ\left(t_{1}, t_{2}, t_{3}, t_{4}, d_{k}^{\prime}\right) \\
&=\left(r_{1} s_{3} t_{3}+r_{2} s_{1} t_{3}+r_{1} s_{4} t_{1}+r_{2} s_{2} t_{1}, r_{1} s_{3} t_{4}+r_{2} s_{1} t_{4}+r_{1} s_{4} t_{2}+r_{2} s_{2} t_{2},\right. \\
&\left.r_{3} s_{3} t_{3}+r_{4} s_{1} t_{3}+r_{3} s_{4} t_{1}+r_{4} s_{2} t_{1}, r_{3} s_{3} t_{4}+r_{4} s_{1} t_{4}+r_{3} s_{4} t_{2}+r_{4} s_{2} t_{2}, d_{k}^{\prime}\right)
\end{aligned}
$$

and

$$
\begin{aligned}
\left(r_{1},\right. & \left.r_{2}, r_{3}, r_{4}, d_{i}^{\prime}\right) \circ\left[\left(\left(s_{1}, s_{2}, s_{3}, s_{4}, d_{j}^{\prime}\right) \circ\left(t_{1}, t_{2}, t_{3}, t_{4}, d_{k}^{\prime}\right)\right]\right. \\
= & \left(r_{1}, r_{2}, r_{3}, r_{4}, d_{i}^{\prime}\right) \circ\left(s_{1} t_{3}+s_{2} t_{1}, s_{1} t_{4}+s_{2} t_{2}, s_{3} t_{3}+s_{4} t_{1}, s_{3} t_{4}+s_{4} t_{2}, d_{k}^{\prime}\right) \\
= & \left(r_{1} s_{3} t_{3}+r_{2} s_{1} t_{3}+r_{1} s_{4} t_{1}+r_{2} s_{2} t_{1}, r_{1} s_{3} t_{4}+r_{2} s_{1} t_{4}+r_{1} s_{4} t_{2}+r_{2} s_{2} t_{2},\right. \\
& \left.r_{3} s_{3} t_{3}+r_{4} s_{1} t_{3}+r_{3} s_{4} t_{1}+r_{4} s_{2} t_{1}, r_{3} s_{3} t_{4}+r_{4} s_{1} t_{4}+r_{3} s_{4} t_{2}+r_{4} s_{2} t_{2}, d_{k}^{\prime}\right) .
\end{aligned}
$$

Since both computation sequences give the same rational form, the associativity holds.

According to Lemma 3.1 , the three substitution operations in $d_{4}^{\prime}=\left(-c_{4} e_{3}, d_{4}, 0,1\right.$, $\left.d_{3}^{\prime}\right) \circ\left(-c_{3} e_{2}, d_{3}, 0,1, d_{2}^{\prime}\right) \circ\left(-c_{2} e_{1}, d_{2}, 0,1, d_{1}^{\prime}\right) \circ\left(d_{1}, 0,1,1, d_{0}^{\prime}\right)$ can be performed in any order. Fig. 1 shows a computation tree for $d_{4}^{\prime}$. The white nodes in the leftmost stage 0 are used for preparing input data only. The black nodes in stages 1 and 2 perform the substitution operation. The single node in stage 3 assigns zero to the pseudo variable $d_{0}^{\prime}$ to obtain the desired result $d_{4}^{\prime}$.

If we combine the computation trees for $d_{i}^{\prime}, 1 \leqslant i \leqslant 8$, we have an overlaid tree network, as shown in Fig. 2, for computing all the suffix values of $d_{8}^{\prime}$. The white nodes in stages 1,2 , and 3 are used for transmitting data only. The pseudo-variables are not real data and hence are omitted there. Note that there are $\log n+2$ stages in this network, meaning that the computation time is only $O(\log n)$. If each node is implemented by a processor, the processor number is as large as $n(\log n+2)$, which is going to be reduced to $\Theta(n / \log n)$ in the later sections.
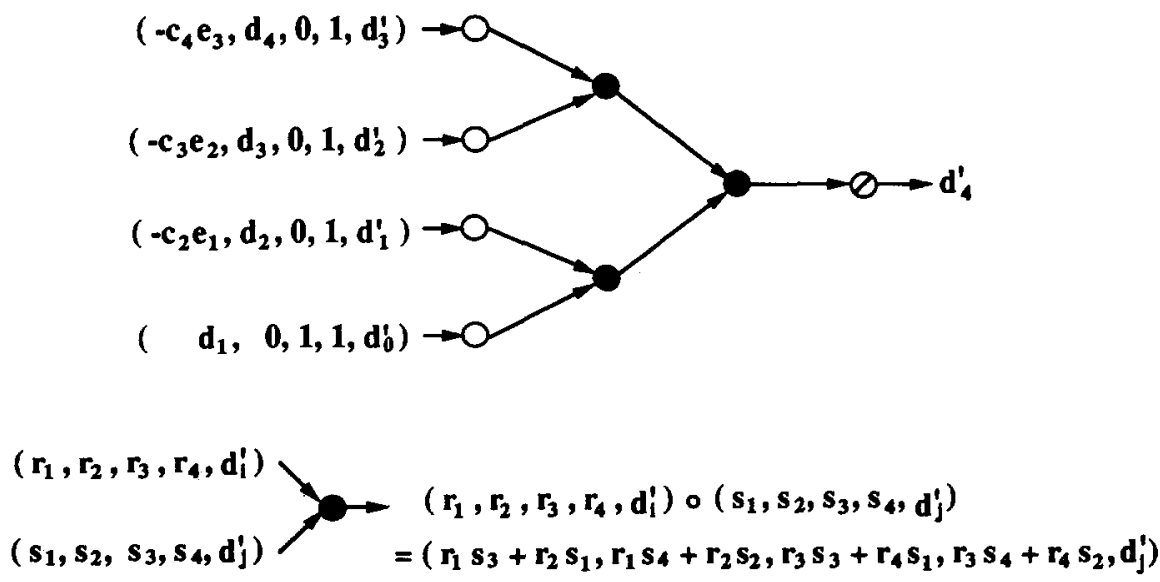

$\left(t_{1}, t_{2}, t_{3}, t_{4}, d_{0}\right) \rightarrow \infty \rightarrow t_{1} / t_{3}$

Fig. 1. A computation tree of $d_{4}^{\prime}$. 


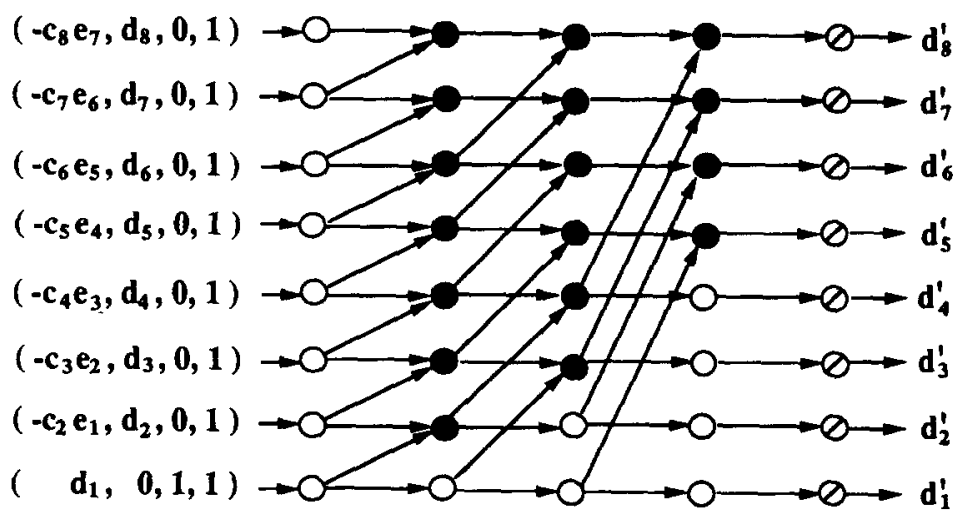

Fig. 2. A network for computing suffix values of $d_{8}^{\prime}$.

Now for the computation of $b_{k}^{\prime}$ in (2.2), the CF for $b_{4}^{\prime} / b_{3}^{\prime}$, say, is given by

$$
\begin{aligned}
X_{4} & =\frac{b_{4}^{\prime}}{b_{3}^{\prime}} \\
& =\frac{b_{4}-\left(c_{4} / d_{3}^{\prime}\right) b_{3}^{\prime}}{b_{3}^{\prime}} \\
& =\left(-c_{4} / d_{3}^{\prime}\right)+\frac{b_{4}}{b_{3}^{\prime}}
\end{aligned}
$$

When $x$ is of the form $r_{1}+r_{2} / r_{3}$, we define $N(x)$ to be the value $r_{1} r_{3}+r_{2}$. The sub_CFs for the above CF are

$$
\begin{aligned}
& X_{4}=\left(-c_{4} / d_{3}^{\prime}\right)+\frac{b_{4}}{N\left(X_{3}\right)}, \\
& X_{3}=\left(-c_{3} / d_{2}^{\prime}\right)+\frac{b_{3}}{N\left(X_{2}\right)}, \\
& X_{2}=\left(-c_{2} / d_{1}^{\prime}\right)+\frac{b_{2}}{N\left(X_{1}\right)}, \\
& X_{1}=\frac{b_{1}}{1+N\left(X_{0}\right)},
\end{aligned}
$$

where $N\left(X_{0}\right)=0, N\left(X_{1}\right)=b_{1}$, and $b_{i}^{\prime}=-\left(c_{i} / d_{i-1}\right) b_{i-1}^{\prime}+b_{i}=N\left(X_{i}\right)$ for all $i$.

Since substituting $\left(s_{1}+s_{2} N\left(X_{j}\right)\right) /\left(s_{3}+s_{4} N\left(X_{j}\right)\right)$ into $X_{i}$ in $\left(r_{1}+r_{2} N\left(X_{i}\right)\right) /\left(r_{3}+r_{4} N\left(X_{i}\right)\right)$, i.e. substituting $s_{1}+s_{2} N\left(X_{j}\right)$ into $N\left(X_{i}\right)$, gives $\left(\left(r_{1}+r_{2} s_{1}\right)+r_{2} s_{2} N\left(X_{j}\right)\right) /\left(\left(r_{3}+r_{4} s_{1}\right)+\right.$ $\left.r_{4} s_{2} N\left(X_{j}\right)\right)$, the substitution operation here is defined as:

$$
\begin{gathered}
\left(r_{1}, r_{2}, r_{3}, r_{4}, N\left(X_{i}\right)\right) \circ\left(s_{1}, s_{2}, s_{3}, s_{4}, N\left(X_{j}\right)\right) \\
=\left(r_{1}+r_{2} s_{1}, r_{2} s_{2}, r_{3}+r_{4} s_{1}, r_{4} s_{2}, N\left(X_{j}\right)\right) .
\end{gathered}
$$

This operation uses 4 multiplications and 2 additions and can be verified to be associative too. The problem of solving the recurrence in (2.2) becomes the suffix computations of $X_{n}$, so the overlaid tree network in Fig. 2 can be used again. 
Similarly, from (2.3), the sub_CFs for $x_{n-3} / x_{n-2}$, say, is given by

$$
\begin{aligned}
& Y_{n-3}=\left(-e_{n-3} / d_{n-3}^{\prime}\right)+\frac{b_{n-3}^{\prime} / d_{n-3}^{\prime}}{N\left(Y_{n-2}\right)}, \\
& Y_{n-2}=\left(-e_{n-2} / d_{n-2}^{\prime}\right)+\frac{b_{n-2}^{\prime} / d_{n-2}^{\prime}}{N\left(Y_{n-1}\right)}, \\
& Y_{n-1}=\left(-e_{n-1} / d_{n-1}^{\prime}\right)+\frac{b_{n-1}^{\prime} / d_{n-1}^{\prime}}{N\left(Y_{n}\right)}, \\
& Y_{n}=\frac{b_{n}^{\prime} / d_{n}^{\prime}}{1+N\left(Y_{0}\right)},
\end{aligned}
$$

where $N\left(Y_{n+1}\right)=0$, and $x_{i}=N\left(Y_{i}\right)$ for all $i$. The problem of solving the recurrence in (2.3) becomes the suffix computations of $Y_{1}$.

\section{Three-phase algorithm on unshuffle network}

In this section, we propose a three-phase algorithm on the unshuffle network for computing all the suffix values of $d_{n}^{\prime}, X_{n}$ and $Y_{1}$. For saving space, we only discuss the suffix computations of $d_{n}^{\prime}$. By adapting appropriate operations in the processors, the suffix computations of $X_{n}$ and $Y_{1}$ can be similarly performed on the same network with the same time complexity. Previously, two approaches have been proposed for some tree-like computations. Based on a depth-width tradeoff in overlaid tree structure, Carlson and Sugla $[2,3]$ presented a perfect shuffle network to solve the first-order linear recurrence in $O(n / p)$ time using $\Theta(p \log p)$ processors. Meijer and Akl [14] presented an optimal binary tree network for computing prefix sums. Our algorithm is based on Stone's unshuffle data routing [19].

In Fig. 2, there is one white node in the first stage, two in the second stage and four in the third stage. The multi-stage unshuffle network which can simulate that network is depicted in Fig. 3, where $S_{i, j}$ and $P_{i, j}, 0 \leqslant i \leqslant 7,1 \leqslant j \leqslant 3$, are simple devices and processors respectively. Let $q_{0} q_{1} \cdots q_{m-1}(m=\log n)$ be the binary representation of $i$. The output line of $P_{i, j}$ is

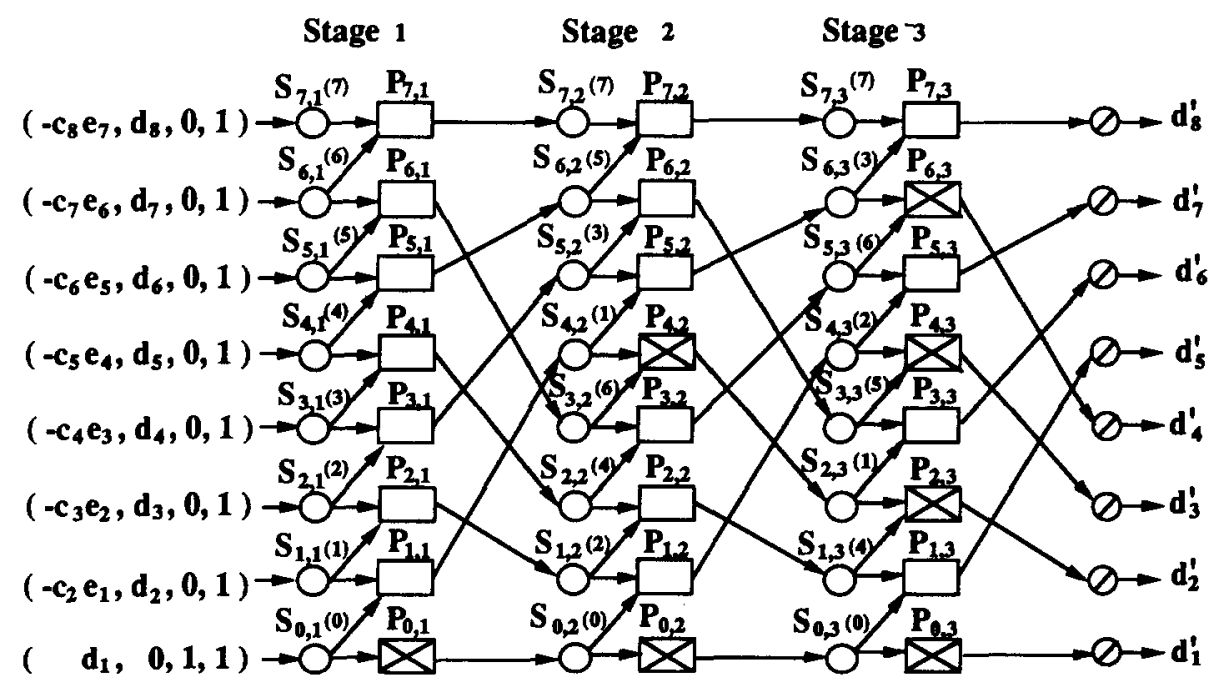

Fig. 3. The multi-stage unshuffle network. 


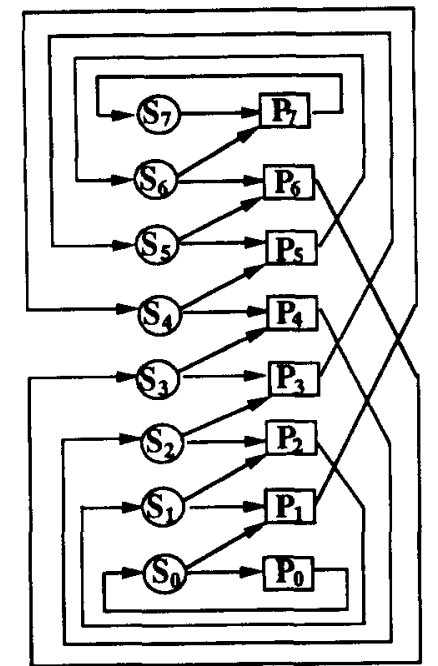

Fig. 4. The single-stage unshuffle network.

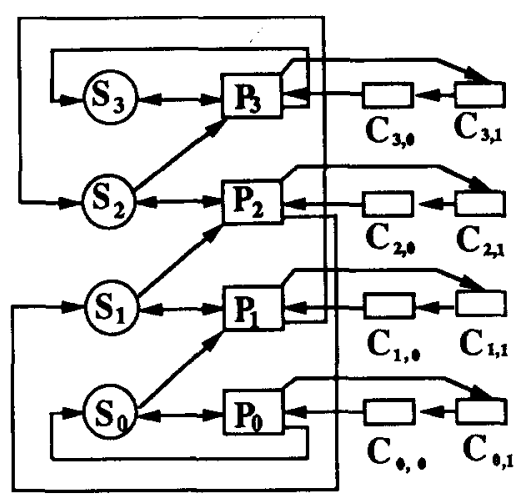

Fig. 5. The three-phase unshuffle network.

connected to $S_{q_{m-1} q_{0} q_{1} \cdots q_{m-2, j+1}}$ to provide the unshuffle data routing. The simple device $s_{i, j}$ is capable of producing two copies of its input and sends them to $P_{i, j}$ and $P_{i+1, j}$ except that the bottom $S_{11 \ldots 1, j}$ has only one receiver. For tracing the messages passed in the network, each simple device is labelled by a number inside the parentheses to indicate where its data originate.

Due to the unshuffle connections from the processors to the simple devices in Fig. 3, the function of white nodes in a stage of Fig. 2 can be controlled by a masking mechanism. For instance, $P_{0,1}$ in stage 1 is masked by a cross mark to merely transmit the input to the next stage; $P_{0,2}$ and $P_{4,2}$ in stage 2 are masked also. Similarly, in stage $3, P_{0.3} . P_{2,3}, P_{4,3}, P_{6,3}$ are masked.

Since all the interconnection patterns between the stages of the multi-stage unshuffle network are the same, we can compress the stages all together to obtain a single-stage unshuffle network, as shown in Fig. 4, which has only $n$ processors. After each iteration of execution, the processors feed the intermediate results back to the simple devices as inputs for the next iteration. The masking on the network at different iterations is exactly what we have described for different stages of the multi-stage network.

In the following, a modified three-phase unshuffle network is proposed to reduce the processor number further. We start with an unshuffle network of $k$ simple devices and $k$ processors, $k \leqslant n$, and change the unidirectional connections from the simple devices to the processors to be bidirectional. To each processor $P_{i}$, attach a serial memory [15], which consists of a linearly connected array of memory cells $C_{i, j}, 0 \leqslant j \leqslant n / k-1$, as depicted in Fig. 5 for $n=8$ and $k=4$. In each execution cycle, the attached memory shifts all its data one position left, $P_{i}$ executes on the data shifted from $C_{i, 0}$ and writes its result to $C_{i, n / k-1}$.

According to the value of the mask register $M_{i}$ resided in $P_{i}$, which is set by the masking mechanism, $P_{i}$ takes one of the following actions:

(1) $M_{i}=0: P_{i}$ transmits the content of its working register $Q_{i}$ to $S_{i}$. (Note that if only one-way connection from $S_{i}$ to $P_{i}$ is allowed, sending data from $P_{i}$ to $S_{i}$ requires $O(\log k)$ time.)

(2) $M_{i}=1: P_{i}$ receives data from $S_{i}$ and transmits it to $S_{q_{m-1} q_{0} q_{1} \ldots q_{m-2}}$, where $m=\log k$.

(3) $M_{i}=2: P_{i}$ performs the substitution operation on its two input data and transmits the result to $S_{q_{m-1} q_{0} q_{1} \ldots q_{m-2}}$. 
(4) $M_{i}=3$ : Except $P_{0}$ which receives input data from $S_{0}, P_{i}$ receives input data from $S_{i-1}$. The routing mechanism is forced to stop and the network goes to another phase of computation.

Initially, $n$ input data (quadruples) are evenly divided into $k$ pipes, each of them is stored in a local memory. Algorithm 4.1 expresses how the network works.

\section{Algorithm 4.1}

Phase 1 (Local computations).

Each $P_{i}$ sequentially computes $n / k$ suffix values from its corresponding pipe of data and stores them in its local memory. The register $Q_{i}$ has the final rational form computed from the pipe of data and transmits it to the register $R_{i}$ in $S_{i}$.

Phase 2 (Macro suffix computations).

All the processors work together using the unshuffle routing mechanism for suffix computations. After $\log k$ steps, the working register $R_{i}$ holds the rational form of $d_{(i+1) n / k}^{\prime}$, for $0 \leqslant i \leqslant k-1$.

Phase 3 (Adaptation).

Each $P_{i}$ except $P_{0}$ receives the rational form from $S_{i-1}$, sequentially modifies all the suffix values calculated in phase 1 by substituting them into the received rational form.

Phase 1 and phase 3 both contain $n / k$ steps. Phase 2 contains $\log k$ steps. So the three-phase algorithm takes $O(n / k+\log k)$ time in total to finish computing all the suffix values of $d_{n}^{\prime}$.

We use $d_{8}^{\prime}\left(=d_{8}+\frac{-c_{8} e_{7}}{d_{7}+} \cdots \frac{-c_{2} e_{1}}{d_{1}}\right)$ as an example to illustrate the algorithm. Let $n=8$ and $k=4$. Initially, the 8 input data (quadruples) for $d_{8}^{\prime}$ are evenly divided into 4 pipes, each containing 2 data. The cells $C_{0,0}$ and $C_{0,1}$ have the data $\left(d_{1}, 0,1,1\right)$ and $\left(-c_{2} e_{1}, d_{2}, 0,1\right)$ respectively. The other cells $C_{i, j} 1 \leqslant i \leqslant 3,0 \leqslant j \leqslant 1$, have the data $\left(-c_{2 i+j+1} e_{2 i+j}, d_{2 i+j+1}, 0,1\right)$. We denote $d_{i}+\frac{-c_{i} e_{i-1}}{d_{i-1}} \cdots \frac{-c_{2} e_{1}}{d_{1}+d_{0}^{\prime}}$ by $F_{i, 1}$ and $d_{i}$ $+\frac{-c_{i} e_{i-1}}{d_{i-1}+} \cdots \frac{-c_{j} e_{j-1}}{d_{j-1}^{\prime}}$ by $F_{i, j}$ for $i \geqslant j \geqslant 2$.

\section{Phase 1.}

Each individual $P_{i}$ computes the two forms $F_{2 i+1,2 i+1}$ and $F_{2 i+2,2 i+1}$ and shifts them back to the serial memory sequentially. That is, $P_{0}$ computes all the suffix values of $F_{2,1} ; P_{1}$ computes all the suffix values of $F_{4,3}$, and so on.

The cell $C_{i, j}$ saves $F_{2 i+j+1,2 i+1}$. After 2 steps in this phase, we have

$$
\begin{array}{ll}
C_{3,0}=F_{7,7}, & C_{3,1}=F_{8,7}, \\
C_{2,0}=F_{5,5}, & C_{2,1}=F_{6,5}, \\
C_{1,0}=F_{3,3}, & C_{1,1}=F_{4,3}, \\
C_{0,0}=F_{1,1}, & C_{0,1}=F_{2,1},
\end{array}
$$

and

$$
Q_{3}=F_{8,7}, \quad Q_{2}=F_{6,5}, \quad Q_{1}=F_{4,3}, \quad Q_{0}=F_{2,1} .
$$

Then, for all $i, M_{i}$ becomes 0 and the content of $Q_{i}$ is transmitted to $R_{i}$.

\section{Phase 2.}

The following macro suffix computations are controlled by the unshuffle routing mechanism. During the first iteration, $M_{0}=1$ and $M_{i}=2$ for $i=1,2,3$. Once the $P_{i}$ 's complete their 
substitution operations, the contents of $R_{i}$ 's become

$$
\begin{aligned}
& R_{3}=F_{8,7} \circ F_{6,5}=F_{8,5}, \\
& R_{2}=F_{6,5} \circ F_{4,3}=F_{6,3}, \\
& R_{1}=F_{4,3} \circ F_{2,1}=F_{4,1}, \\
& R_{0}=F_{2,1} .
\end{aligned}
$$

During the second iteration, $M_{i}=2$ for all $i$ except $i \equiv 0 \bmod 2$ for which $M_{i}=1$. Once the $P_{i}$ 's complete their substitution operations again, the contents of $R_{i}$ 's become

$$
\begin{aligned}
& R_{3}=F_{8,5} \circ F_{4,1}=F_{8,1}, \\
& R_{2}=F_{6,3} \circ F_{2,1}=F_{6,1}, \\
& R_{1}=F_{4,1}, \\
& R_{0}=F_{2,1} .
\end{aligned}
$$

Let $k$ be $2^{h}$. In general, during the $j$ th iteration, $1 \leqslant j \leqslant h, M_{i}=2$ for all $i$, except $i \equiv 0 \bmod \left(2^{h+1-j}\right)$ for which $M_{i}=1$. After this phase of computation, $R_{i}$ saves $F_{2(i+1), 1}$, a macro suffix value.

\section{Phase 3.}

During the first step of this phase, $M_{i}=3$, each $P_{i}$ except $P_{0}$ receives input data from $S_{i-1}$. The contents of $Q_{i}$ 's become

$$
Q_{3}=F_{6,1}, \quad Q_{2}=F_{4,1}, \quad Q_{1}=F_{2,1}, \quad Q_{0}=F_{2,1} .
$$

The unshuffle routing mechanism is forced to stop and each $P_{i}$ sequentially modifies the contents of $C_{i, j}$ 's, $j=0,1$, by performing the substitution operation $Q_{i} \circ C_{i, j}$. The final results are

$$
\begin{array}{ll}
C_{3,0}=F_{7,7} \circ F_{6,1}=F_{7,1}, & C_{3,1}=F_{8,7} \circ F_{6,1}=F_{8,1}, \\
C_{2,0}=F_{5,5} \circ F_{4,1}=F_{5,1} & C_{2,1}=F_{6,5} \circ F_{4,1}=F_{6,1}, \\
C_{1,0}=F_{3,3} \circ F_{2,1}=F_{3,1} & C_{1,1}=F_{4,3} \circ F_{2,1}=F_{4,1}, \\
C_{0,0}=F_{1,1}, & C_{0,1}=F_{2,1} .
\end{array}
$$

\section{Cost optimality}

As discussed in Section 4, all the suffix values of $d_{n}^{\prime}$ can be computed in $O(n / k+\log k)$ time using $k$ processors. For the subsequent parts of the computation, namely computing the suffix values of $X_{n}$ and $Y_{1}$, the algorithm works as well if we properly adjust the substitution operations in the processors. Preparing the input data from one part of the computation to another takes $O(n / k)$ time, $n / k$ is the size of each data pipe. So we have the following lemma.

Lemma 5.1. A tridiagonal system can be solved in $O(n / k+\log k)$ time on an unshuffle network of $k$ processors.

The performance of a parallel algorithm can be measured by

$$
\text { Cost }=\text { Processor Number } \times \text { Execution Time. }
$$

Given a problem, if the cost of a parallel algorithm matches the sequential time lower bound within a constant, the parallel algorithm is said to be cost-optimal. In the case of solving the 
tridiagonal system, since there are $n$ values to be produced, the sequential time lower bound is clearly $\Omega(n)$.

Theorem 5.1 determines the number of processors that is required to minimize both the cost and the execution time.

Theorem 5.1. For solving tridiagonal system on the unshuffle network, we can use $\Theta(n / \log n)$ processors to achieve the optimal cost $\Omega(n)$ and the computing time $O(\log n)$.

Proof. By Lemma 5.1, the cost of our three-phase computation model with $k\left(=2^{h}\right)$ processors is $O(k(n / k+\log k))=O(n+k \log k)$. Because $k \log k$ is an increasing function of $k$, the solution $k$ satisfying $k \log k=c n$ (for some constant $c$ ) is the number of processors needed at most. The above equation can be written as

$$
2^{h} h=c n=n^{\prime} \text {. }
$$

For applying the bootstrapping method [8], we initially let

$$
h=\log n^{\prime}-\log h \text {. }
$$

Therefore,

$$
h=O\left(\log n^{\prime}\right) .
$$

Substituting this approximation into (5.1) yields the following better result:

$$
h=\log n^{\prime}+O\left(\log \log n^{\prime}\right) .
$$

Substituting this approximation again into (5.1) improves the result as follows:

$$
\begin{aligned}
h & =\log n^{\prime}-\log \left(\log n^{\prime}\left(1+O\left(\frac{\log \log n^{\prime}}{\log n^{\prime}}\right)\right)\right) \\
& =\log n^{\prime}-\log \log n^{\prime}-\log \left(1+O\left(\frac{\log \log n^{\prime}}{\log n^{\prime}}\right)\right) .
\end{aligned}
$$

By Taylor's expansion, we know

$$
e^{\frac{\log \log n^{\prime}}{\log n^{\prime}}}=1+O\left(\frac{\log \log n^{\prime}}{\log n^{\prime}}\right) .
$$

That is,

$$
\frac{\log \log n^{\prime}}{\log n^{\prime}}=\log \left(1+O\left(\frac{\log \log n^{\prime}}{\log n^{\prime}}\right)\right) .
$$

Hence, (5.2) can be written as

$$
h=\log \frac{n^{\prime}}{\log n^{\prime}}+O\left(\frac{\log \log n^{\prime}}{\log n^{\prime}}\right) .
$$

Recovering $k$ from $k=2^{h}$,

$$
\begin{aligned}
k & =\frac{n^{\prime}}{\log n}+O\left(2^{\frac{\log \log n^{\prime}}{\log n^{\prime}}}\right) \\
& =\Theta(n / \log n) .
\end{aligned}
$$

It is clear that when $k=\Theta(n / \log n)$ processors are used in the network, the computing time is $O(n / k+\log k)=O(\log n)$. 


\section{Concluding remarks}

In this paper we have first transformed the solution of a tridiagonal system into three recurrence equations. The recursive-doubling method $[12,17]$ is an alternative approach to solve these recurrence equations through a rather tricky divide-and-conquer reformulation of the recurrences into ones which use two indices. Our approach is simply doing their CF expansions and applying the straightforward substitution concept. Not only the associativity of the substitution brings out the parallelism for the computation, but also it supplies the idea of three-phase computation to reduce the processor number. An analysis technique presented in [6] can be used to verify that our method is numerically stable when the coefficient matrix $A$ is symmetric and diagonally dominant.

The substitution scheme used in this paper was originally presented in [5] for the parallel computation of general continued fractions. It allows all the first $n$ prefix values of any continued fraction to be computed in $O(\log n)$ time using $\Theta(n / \log n)$ processors. We also embed a self-substitution concept into the scheme to derive a sequential $O(\log n)$ algorithm to fast compute periodic CFs [4]. Its applications include the approximation of quadratic surd numbers and the solving of second-order linear recurrences with constant coefficients.

\section{References}

[1] D.P. Bertsekas and J.N. Tsitsiklis, Parallel and Distributed Computation: Numerical Methods (Prentice-Hall, Englewood Cliffs, NJ, 1989).

[2] D.A. Carlson and B. Sugla, Time and processor efficient parallel algorithms for recurrence equations and related problems, in: Proc. 1984 Internat. Conf. Parallel Processing, IEEE Computer Society Press, Washington DC (1984) $310-314$.

[3] D.A. Carlson and B. Sugla, Adapting shuffle-exchange like parallel processing organizations to work as systolic arrays, Parallel Comput. 11 (1) (1989) 93-106.

[4] K.L. Chung, W.C. Chen and F.C. Lin, Fast computation of periodic continued fractions, Information Processing Letters 33 (2) (1989) 67-72.

[5] K.L. Chung, F.C. Lin and W.C. Chen, Parallel computation of continued fractions, submitted.

[6] P. Dubois and G. Rodrigue, An analysis of recrusive doubling algorithm, in: High Speed Computer and Algorithm Organization, D.J. Kuck, D.H. Lawrie and A.H. Sameh eds. (Academic Press, New York, 1977).

[7] G.H. Golub and C.F. Van Loan, Matrix Computations (Johns Hopkins University Press, Baltimore, MD, 1983).

[8] D.H. Greene and D.E. Knuth, Mathematics for the Analysis of Algorithms (Birkhäuser, Boston, 2nd ed., 1982).

[9] D. Heller, Some aspects of the cyclic reduction algorithm for block tridiagonal linear systems, SIAM J. Numer. Anal. 13 (4) (1976) 484-496.

[10] R.W. Hockney, A fast direct solution of poisson's equation using fourier analysis, J. ACM 12 (1) (1965) 95-113.

[11] S.L. Johnsson, Solving tridiagonal systems on ensemble architectures, SIAM J. Sci. Stat. Comput. 8 (3) (1987) 354-392.

[12] P.M. Kogge and H.S. Stone, A parallel algorithm for the efficient solution of a general class of recurrence equations, IEEE Trans. Comput. C-22 (8) (1973) 786-793.

[13] J.J. Lambiotte, Jr. and R.G. Voigt, The solution of tridiagonal linear systems on the CDC STAR-100 computer, ACM Trans. Math. Software 1 (4) (1975) 308-329.

[14] H. Meijer and S.G. Akl, Optimal computation of prefix sums on a binary tree of processors, Internat. J. Parallel Programming 16 (2) (1987) 127-136.

[15] R.M. Owens and J. Ja'Ja', Parallel sorting with serial memories, IEEE Trans. Comput. C-34 (4) (1985) 379-383.

[16] A.H. Sameh and D.J. Kuck, A parallel QR algorithm for symmetric tridiagonal matrices, IEEE Trans. Comput. C-26 (2) (1977) 147-153.

[17] H.S. Stone, An efficient parallel algorithm for the solution of a tridiagonal linear system of equations, J.ACM 20 (1) (1973) 27-38.

[18] H.S. Stone, Parallel tridiagonal equation solvers, ACM Trans. Math. Software 1 (4) (1975) 289-307.

[19] H.S. Stone, Introduction to Computer Architecture (Science Research, Chicago, IL, 1980).

[20] H.H. Wang, A parallel method for tridiagonal equations, ACM Trans. Math. Software 7 (2) (1981) 170-183. 